\title{
Estimation of Rice Plant Height from a Low-Cost UAV- Based Lidar Point Clouds
}

\author{
Phan, A. T. T. ${ }^{1,2^{*}}$ and Takahashi, $\mathrm{K}^{3}$ \\ ${ }^{1}$ Department of Geomatics Engineering, Faculty of Civil Engineering, Ho Chi Minh City University of \\ Technology, 268 Ly Thuong Kiet Street, District 10, Ho Chi Minh City, Vietnam \\ E-mail: ptathu@hcmut.edu.vn \\ ${ }^{2}$ Vietnam National University Ho Chi Minh City, Linh Trung Ward, Thu Duc District, Ho Chi Minh City, \\ Vietnam \\ ${ }^{3}$ Nagaoka University of Technology, Environmental Civil Engineering Department, 1603-1, Kami-Tomioka, \\ Nagaoka, Niigata 940-2188, Japan, E-mail: ktakaha@nagaokaut.ac.jp \\ *Correspondence Auhtor
}

\begin{abstract}
UAV systems are considered effective tools to collect information regarding crops. In this study, the rice growth was observed by a small UAV-based LiDAR system from above. For developing the system, DJI S800 was chosen as a platform on which a non- survey-grade laser scanner HOKUYO UTM3OLX-EW was mounted. Field experiments were carried out from late June to late early August 2017 in Nagaoka city, Niigata Prefecture, Japan. Percentile analysis is applied to locate the top and bottom positions of rice plants in three targeted areas. LIDAR-derived plant height is computed by taking the difference between the bottom and the rice plant's top. As a result, the LiDAR-derived canopy height well correlates to rice plant height $\left(R^{2} \geq 0.86 ; R M S E<6.0 \mathrm{~cm}\right)$. The small root means square error $(R M S E=4.9 \mathrm{~cm})$ is achieved with area 3. In the general case, the RMSE is $5.5 \mathrm{~cm}\left(R^{2}=0.88\right)$. These results illustrate the capability of estimate plant height before the heading stage from UAV-based LiDAR point clouds without ground surface detection.
\end{abstract}

\section{Introduction}

Over the past decades, rice growth has been validated directly on the field based on the value of the rice plant's physical parameters such as plant height, the number of stems, leaves colors (Yamamoto et al., 1994). From these results, the farmers give their decision for controlling the rice growth based on their experiments. In general, these mentioned parameters are measured periodically to ensure the rice plant grows well. In detail, rice leaf color indicates rice leaf nitrogen content (Singh et al., 2012 and Wang et al., 2013). Plant height and stem number change over time and enormously increase in the vegetation phase of the rice plant growth. The number of rice plant stems is the first component of rice yield. With the grain weight and spikelet per panicle, the rice plant stem number determines the rice yield (Wang et al., 2007 and Badshah et al., 2014). The rice plant height indicates the rice plant growth, which is also a common factor studied to monitor the rice growth rate. Both rice plant height and stem number determine the rice yield ( Wang et al., 2007, Badshah et al., 2014, Thomas et al., 1994, Fernandez et al., 2009; Bai et al., 2011). Up to date, rice growth has been validated by counting the number of rice leaves, measuring the leaf area index, fresh and dry weight, and crop biomass (Dobermann and Pampolino 1995, Takai et al., 2006, Hasanuzzaman et al., 2010 and Bendig et al., 2014). However, collecting this information by fieldwork is a labor-intensive task. The recording and storage of collected data sometimes would be subject to unintentional mistakes due to technicians' carelessness.

Today, remote sensing is considered an effective method to quickly and accurately collect the entire field's data or only on-demand parts of the area, but fewer labor costs (Atzberger, 2013 and Willers, 2012). In detail, vegetation indices derived from optical satellite images are used for cropland identification and yield forecasting (Tennakoon et al., 1992 and Fablo and Rembold, 2001). Radar images have also been popular used for monitoring rice crops by exploiting the high backscatter increase at the beginning of the growing cycle (Shao et al., 2001 and Bouvet and Le Toan, 2011). However, removing the effect of cloud cover is the biggest problem of using optical satellite images. Biophysical rice plant variables have also been obtained from the SAR sensor's backscattering coefficients (Koppe et al., 2013 and Inoue et al., 
2014). In general, using satellite images, we can get the information on a large scale. Their spatial resolutions are selected depending on the size of the target object and each study's purpose. In several meters, the normal resolution is good enough for monitoring crop growth differences among plots or fields. However, in precision agriculture, the difference in crop growth inside each plot is focused. In such a situation, the spatial resolution and long revisit period of existed satellite image and are not enough for the analysis. Photogrammetry is also suggested for monitoring the crop growth information. However, it is costly to obtain up-todate images; the received data quality is unstable, the data processing is complicated. Low altitude remote sensing (LARS) systems or UAV-based remote sensing systems are developed for many purposes, including rice crop monitoring (Swain and Zaman, 2012). The nitrogen nutrition status of rice plants has been estimated from color digital images (Li et al., 2010 and Lee, 2013). Besides nitrogen status, digital camera-derived vegetation indices have been used for monitoring seasonal rice growth in quantitative (Sakamoto et al., 2011). However, rice plant height and the number of stems are not easy to obtain from digital camera photos. In general, each method has both advantages and disadvantages. Based on the specific purpose of each study, the suitable method is selected.

The laser scanning technology has been a valuable method for collecting dense and accurate spatial data in a short time (Lichti et al., 2012). This technique can be applied for monitoring forests or small plants of crops. In the most application, laser scanning data are usually classified to generate the high accuracy digital surface model, digital elevation model with high resolution (Mcintosh et al., 2000 and Ma, 2005), or estimating canopy height (Wang and Glenn, 2008, Huang et al., 2009, Zawawi et al., 2005, Sibona et al., 2017, Ehlert and Heisig, 2005 and Ehlert et al., 2006 and 2010 ), canopy structure (Rice et al., 2005), carbon stork (Maan et al., 2005) and vertical plant density profile (Hosoi et al., 2006 and 2009). The terrestrial laser scanner (TLS) can provide a fast and accurate procedure to collect the rice crops information (Tilly et al., 2004, Kaizu et al., 2012 and Zhang and Grift, 2012), but this is expensive equipment. The ground surface position or another reference surface close to the ground were required for computing plant height. Unfortunately, such a surface cannot always be determined, especially in wetland paddies or densely packed crops. The incident angle affects the obtained point clouds. Besides, for dense rice fields, the accessibility of laser pulses to the rear trees is limited because the previous rice plants themselves obscure them. To solve the mentioned problems of TLS, we proposed a method for rice plant height estimation using LiDAR measurements above rice canopy without the ground surface detection (Phan et al., 2016). This method was evaluated based on 3D point cloud data analysis in a small area that was acquired by a LiDAR sensor above rice plants. In the mentioned paper, SICK and Hokuyo UTM 30LX were mounted on a fixed frame at the height of $3 \mathrm{~m}$ from the paddy surface to observe the rice plant's small area. A UAV- based laser scanner system is considered as a useful tool to quickly collect 3D point cloud data of rice plants in a wide area. In this study, preliminary research is carried out to evaluate our proposed method's applicability in a wide area based on an analysis of $3 \mathrm{D}$ point cloud data of rice plants collected by a UAV-base LiDAR system at a low altitude (3 to 5 meters). For reducing the cost of equipment, the developed system is assembled by a low-cost UAV and a nonsurvey grade LiDAR sensor.

\section{Materials and Methods}

\subsection{The Developed UAV-based LiDAR System}

For developing the system, all devices are selected under low-cost, compact, and light-weight requirements. In detail, the chosen platform is a hexrotor aircraft, DJI spreading wings S800 with a maximum of $2.5 \mathrm{~kg}$ of payload. DJI Wookong-M controls this UAV, a popular autopilot with $0.5 \mathrm{~m}$ and $2 \mathrm{~m}$ accuracy in vertical and horizontal, respectively. The UTM 30 LX- EW laser scanner, known as a LiDAR sensor, is fixed in a vertically downward direction. This device is mounted on a 3axis gimbal (TAROT 5D) to maintain its horizontal attitude. The Sensor-Com Kit recorded data at a rate of $5 \mathrm{~Hz}$ for locating the position of the UAV system. Sensor controlling and data recording is performed by Raspberry Pi 2 . A GoPro Hero3 was installed in front of the UAV itself to record a scene under the UAV. Finally, the whole system is supplied the power by Pin Lithium Polymer (LiPo) for capable of flight times of about 8 minutes. The developed system and its devices are clearly illustrated in Figure 1.

\subsection{Data Acquisition}

For setting up the test field, a field in Nagaoka city, Niigata Prefecture, was targeted in this study. For establishing the test field, the Koshihikari rice variety was transplanted in the targeted field on May 31, 2017. Planting density was 16.7 plants. $\mathrm{m}^{-2}$ with planting geometry of $30 \mathrm{~cm} \times 20 \mathrm{~cm}$. A missing plant area occurred due to using a transplanting machine. Ten reflectors were placed in the field to identify the target area easily on 3D point cloud data 
acquired by the UAV-LiDAR system (Figure 2). Reflector positions were also used as GCPs for generating 3D point cloud data. Two reflectors at the east and west ends were used as landmarks for the flight's start and end points. To collect the data, field experiments were carried out during the vegetation phase of the rice plant from late June to late July 2017. The flight path was set along the centerline of the target area. The UAV system flew over the target field under automatic control mode at the set altitude of $5 \mathrm{~m}$ above from the paddy surface to collect the rice field data below.
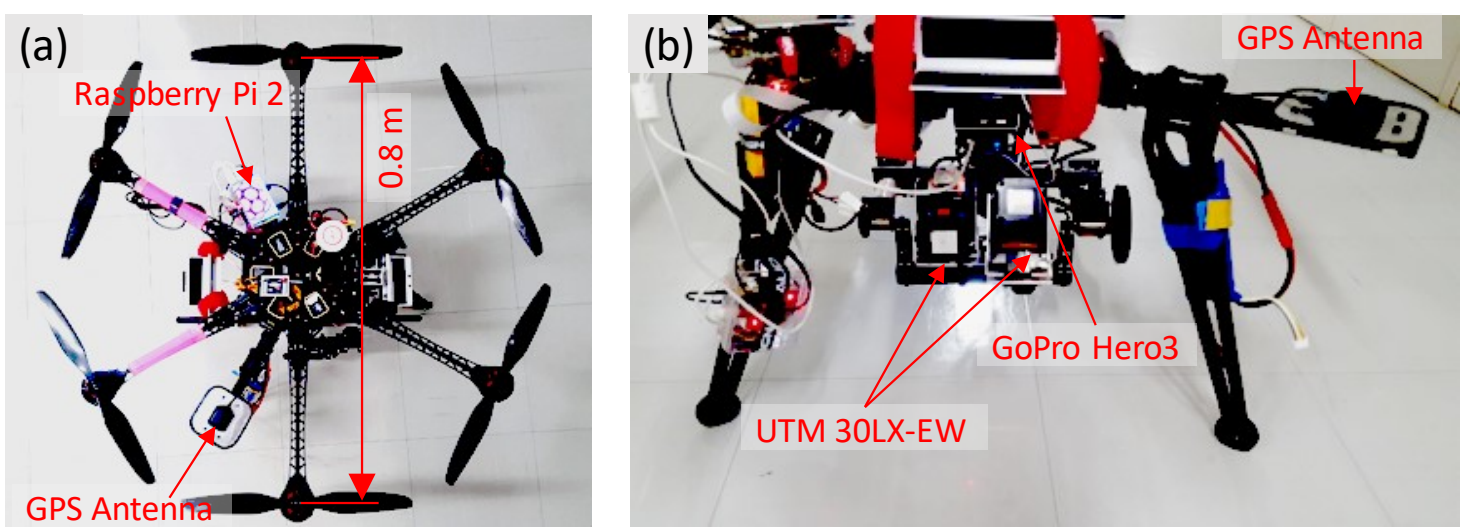

Figure 1: Developed UAV-based laser scanner system (a) top view and (b) front view

Table 1: Specifications of UTM 30LX-EW

\begin{tabular}{|l|l|}
\hline Wavelength & $905 \mathrm{~nm}$ \\
\hline Field of view & 270 degrees \\
\hline Angular resolution & 0.25 degrees \\
\hline Scan speed & $25 \mathrm{msec}$ \\
\hline Accuracy & $\pm 30 \mathrm{~mm}(<10 \mathrm{~m})$ \\
\hline
\end{tabular}

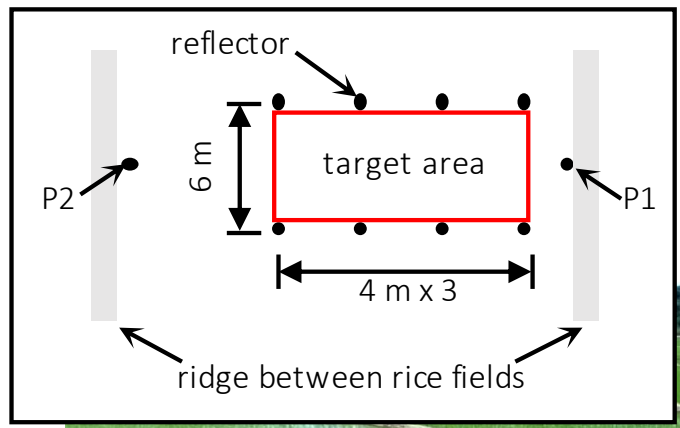

\section{UAV-LiDAR system}
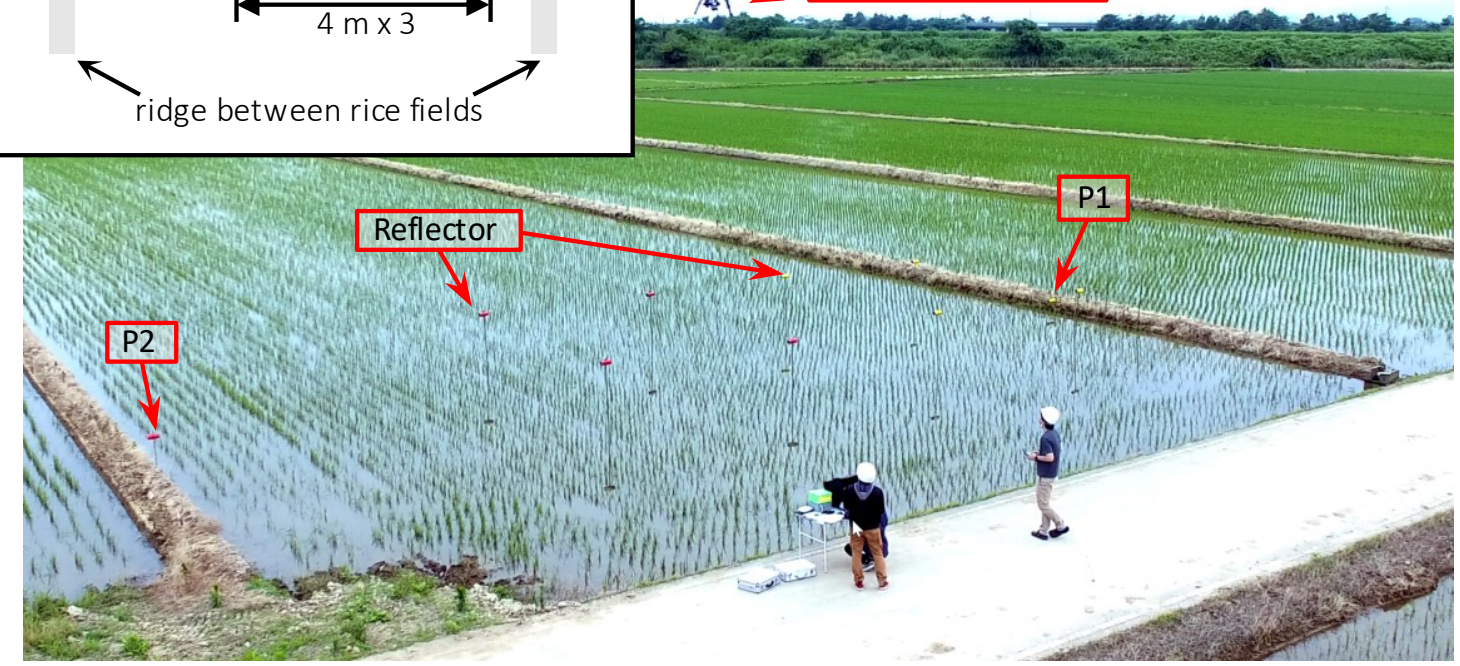

Figure 2: Ten reflectors are arranged on the experimental site to locate the position of target areas 
The low flight altitude was set up to make sure that the rice leaves can be observed. The intensity raw image in which UTM 30LX-EW data was directly arranged, exported, and checked right after completing the experiment to ensure the quality of collected data. During the experimental period, the rice plant was almost stand up straight. It was expected that the top of rice plant could be observed by LiDAR sensor in a windless situation in such a condition. After completing each experiment, rice plant height and stem number were measured directly for the validation data. As a result, eight experiments had been performed in total. The reflectors were recognized on the intensity raw image. However, the arrangement of those was deformed in each observation. It has occurred that the flight paths (altitude and horizontal position) were unstable due to the flight performance limit of S800 and Wookong-M. The flight altitude was different in each observation and varied from 4 to 7 (m). Therefore, the collected data must be corrected for further analysis.

\subsection{Methodology}

\subsubsection{Generation of $3 D$ point clouds}

For generating 3D point clouds, the UAV position was first calculated by the post kinematic positioning from data recorded by a single frequency GPS receiver. It was assumed that the laser scanning plane is vertical, and the average vector of the laser scanning plane is matched to the flight direction (the nose direction). For each scanning point in any scanning plane (Figure 3a-b), its coordinates in the UTM30 LX-EW object coordinate system (LIDAR sensor coordinate system) are expressed as equation (1).

$$
\left(\begin{array}{l}
x_{L} \\
y_{L} \\
z_{L}
\end{array}\right)=\left(\begin{array}{c}
-r \cdot \sin \theta \\
0 \\
-r \cdot \cos \theta
\end{array}\right)
$$

Equation 1

(a)

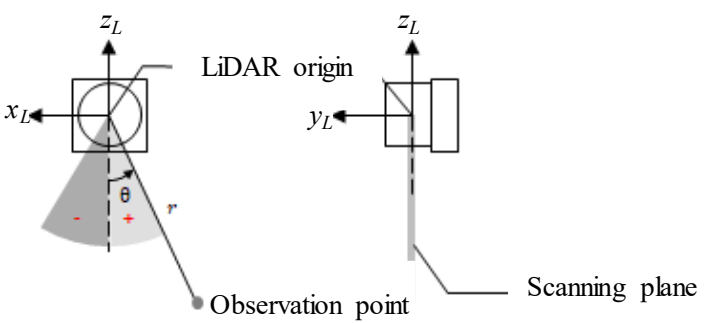

Where:

$\boldsymbol{P}_{\text {UTM }}=\left(\begin{array}{llll}x_{L}, & y_{L}, & z_{L}\end{array}\right)^{T}$ : The coordinate of scanning point in LiDAR sensor coordinate system

$r$ : Observed range

$\theta$ : Scanning angle

Then, the position of the UAV $\left(\boldsymbol{P}_{U A V}=\right.$ $\left.\left(x_{\text {uav }}, y_{\text {uav }}, z_{\text {uav }}\right)^{T}\right)$ is calculating from GPS data by applying the post kinematic positioning method. The relationship between the origin of the LiDAR sensor and the $8^{\text {th }}$ of the Japan orthogonal coordinate system is displayed in Figure 3. The coordinate of all scanning points in the LIDAR sensor coordinate system is transformed to the $8^{\text {th }}$ of the Japan orthogonal coordinate system, using equation (2).

$$
\boldsymbol{P}=\boldsymbol{R}_{Z} \boldsymbol{P}_{U T M}+\boldsymbol{T}+\boldsymbol{P}_{U A V}
$$

Equation 2

Where:

$\boldsymbol{P}=(x, y, z)^{T}$ is the coordinate of scanning point in the $8^{\text {th }}$ of the Japan orthogonal coordinate system.

$\boldsymbol{T}=\left(x_{t}, y_{t}, z_{t}\right)^{T}:$ is the offset between the origin of the UTM30 LX-EW and the GPS antenna position.

$\boldsymbol{P}_{U A V}=\left(x_{\text {uav }}, y_{\text {uav }}, z_{\text {uav }}\right)^{T}$ : imply the position of UAV in the $8^{\text {th }}$ of the Japan orthogonal coordinate system. The $\boldsymbol{R}_{z}$ is written by equation (3) with the counterclockwise rotation angle $\alpha$.

$$
\boldsymbol{R}_{z}=\left(\begin{array}{ccc}
\cos \alpha & \sin \alpha & 0 \\
-\sin \alpha & \cos \alpha & 0 \\
0 & 0 & 1
\end{array}\right)
$$

Equation 3

However, the developed UAV-LiDAR system did not include the recording nose direction sensor. Additionally, the UAV position data calculated by the post kinematic positioning in this study using the RTKLIB were almost in RTK float.

Figure 3: Illustration of the relationship between LiDAR sensor's origin and the 8th of the Japan orthogonal coordinate system. (a) X-Z plane and (b) Y-Z plane in LiDAR sensor coordinate system, (c) The position of LIDAR sensor origin and GPS antenna are different, and (d) the nose direction of UAV. 
Therefore, the 3D point cloud must be corrected. For this, the nose direction was assumed unchanged over the target area. A tentative 3D point cloud was generated with zero degrees of nose direction $(\alpha=0)$. Then, the point cloud's horizontal coordinates were corrected by applying the Affine transformation based on the horizontal coordinates of markers. Finally, the mean value of markers' vertical coordinates was used to convert the vertical coordinate of the $3 \mathrm{D}$ point cloud.

\subsubsection{LiDAR-derived canopy height}

The coordinates of target area corners are identified by offsetting all reflectors' coordinates to the center at a distance of $0.5 \mathrm{~m}$. In this way, the reflectors are completely removed from the extracted data. The data of three target areas in each experiment are extracted. The plant height estimation method developed in our previous study (Phan et al., 2016) is applied to the vertical distance data acquired by the developed UAV- based LiDAR system. Phan et al. (2016) clarified the relationship between LiDARderived rice plant height and the rice plant height directly measured on the field before the heading stage (from June to late July). To apply our previous method, it is necessary to locate the rice plant's top and bottom positions by using percentile analysis. According to our previous study (Phan et al., 2016), the top and bottom of rice plant height are identified at $1^{\text {st }}$ and $95^{\text {th }}$ percentile rank, respectively. Finally, LiDAR-derived canopy height is calculated by taking the difference between the bottom and the rice plant's top (equation 4). In this way, the ground surface had not detected. To reduce the effect of ununiformed rice growth in a large area, each target area is divided into many sample areas with $0.5 \mathrm{~m}$ of spatial resolution., the LiDAR-derived canopy height computing process applies to all samples. The LiDAR-derived canopy height represents for each target area is the average LiDAR-derived canopy height all samples locate inside it.

$$
H^{\text {laser }}=D^{95}-D^{1}
$$

Equation 4

Where:

$H^{\text {laser }}$ : LiDAR-derived rice plant height

$D^{95}$ : the value of vertical distance corresponding to the 95th percentile rank

$D^{1}$ : the value of vertical distance corresponding to the 1 st percentile rank

\section{Results}

\subsection{Manual Measurement Results}

As mentioned above, the rice plant height and stem number are manually collected in each experiment. In total, there are nine measurements performed during the observed period. During the experimental period, rice plant height and number of stems increases rapidly. Their changes are displayed in Figure 4. In detail, rice plant height linearly increases from 30 to $86(\mathrm{~cm})$. The number of stems varies from approximately 160 stems. $\mathrm{m}^{-2}$ to about 470 stems. $\mathrm{m}^{-2}$ and reaches the maximum value on July 21， 2017 (approximate 50 days after transplanting). In general, the standard deviation of average plant height and stem number is less than 2 $\mathrm{cm}$ and approximate 30 stems. $\mathrm{m}^{-2}$ for three target areas. As a result, there is an insignificant difference between the value of plant height and the number of stems in the three target areas. As a result, the rice grown in the test field is assumed uniform.

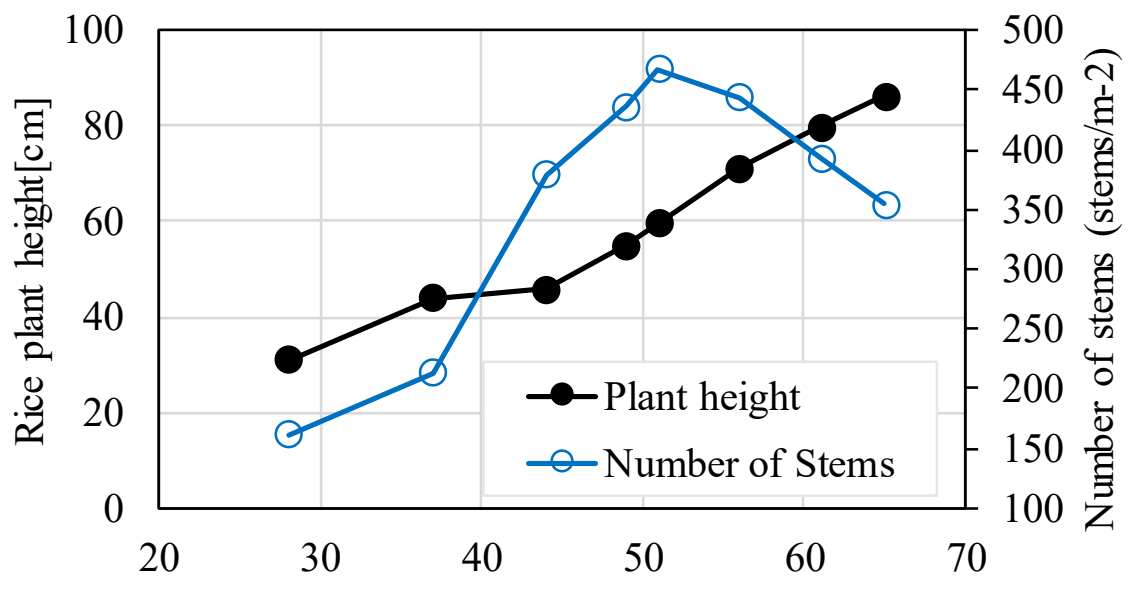

Day after transplanting (DAT)

Figure 4: The average value of rice plant height and number of stems in three target areas 
.2 Relationship between LiDAR-Derived Canopy

Height and Plant Height

According to Figure 5, the distributions of scanning points in three areas are similar and changed over time. In the first observation, the paddy was covered by water, and most laser pulses could not reach the ground surface. Therefore, the histogram peak implied the level of the water surface. As a result, it was higher than the histogram peak in the second observation. The down-skew distribution in the second observation implies that the rice plant was small and could not cover the ground surface; therefore, large laser pulses reach the ground surface.

Along with the growing up of rice plant, the number of laser pulses reaching the ground surface decreases because the rice plant itself hides the ground surface, and the histogram peak was moving up. Besides, the calculated LiDAR-derived canopy height in three areas is an insignificant difference (Figure 6). This result shows that the LiDAR-

derived canopy heights are matched to the rice growth conditions in three test areas. The decrease of LiDAR-derived canopy height in the last observation on August 4 (65 DAT) might be caused by the downward of rice leaves. This phenomenon will affect the relationship between LiDAR-derived canopy height and plant height in this area. The correlation between LiDAR-derived canopy height and plant height is displayed in Figure 7 when LiDAR-derived canopy height is plotted against plant height. The regression lines show a high coefficient of determination $\left(\mathrm{R}^{2} \geq 0.82\right)$. This relationship is expressed by a formula applied for estimating plant height from point cloud data. To evaluate results, estimated rice plant heights are compared with measured plant height. The small root means square error $(\mathrm{RMSE}=7.7 \mathrm{~cm})$ is achieved in the general case. The smallest RMSE is achieved in area $3(\mathrm{RMSE}=6.0 \mathrm{~cm})$. These results illustrate the capability of estimate plant height form UAV- based LiDAR point clouds

(e)

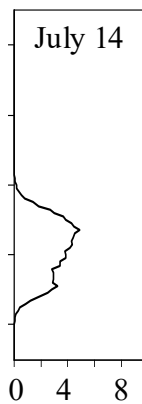

(d)

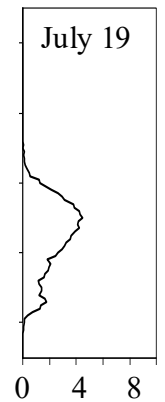

(f)

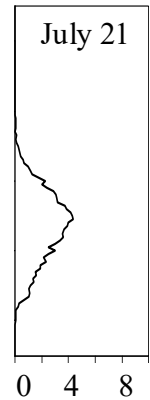

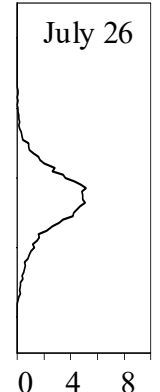

(g)

(h)

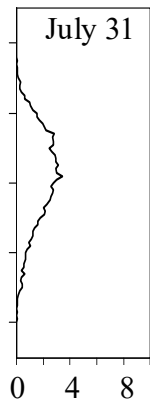

Relative density (\%)

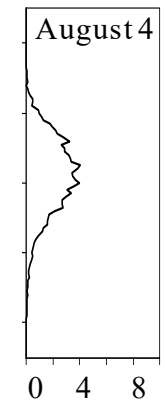

Figure 5: The histogram of the point cloud in target area 2. The red dash lines show the position of the histogram peaks in the two first observations. The histogram peak in the first observation implied the level of water surface, and it was higher than the histogram peak in the second observation

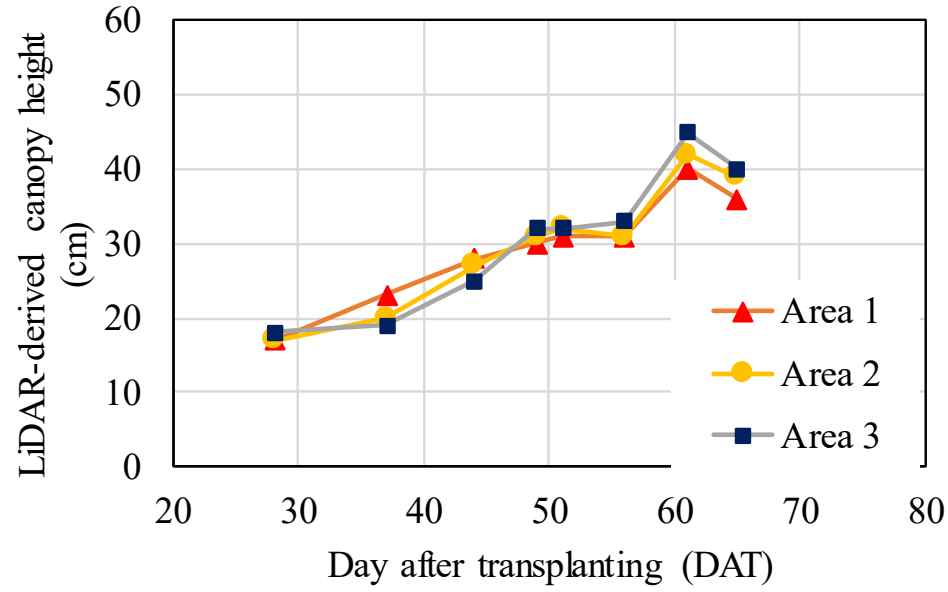

Figure 6: The value of LiDAR- Derived canopy height in three areas 
(a)

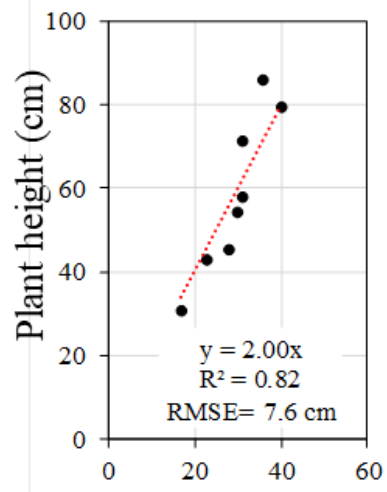

(b)

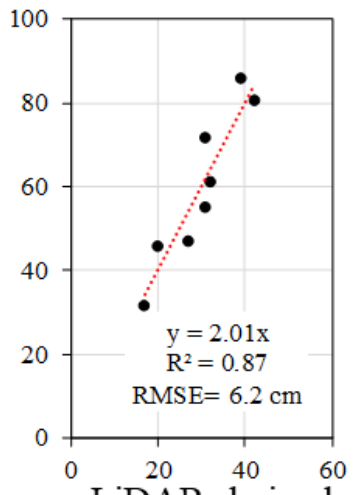

(c)

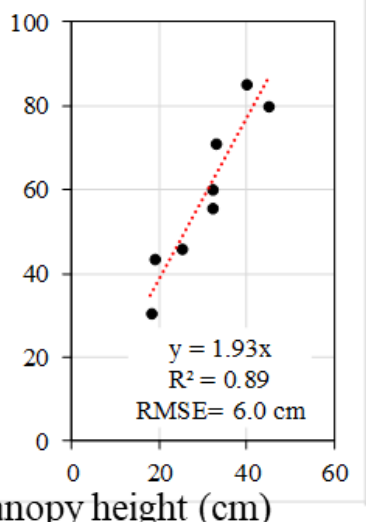

(d)

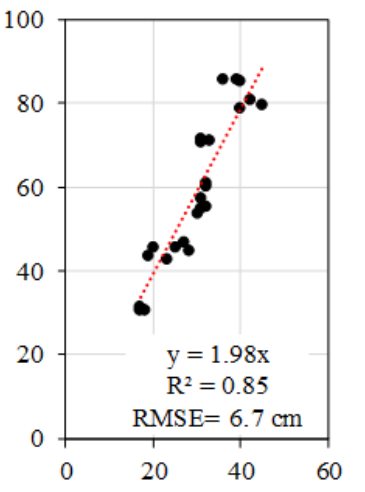

Figure 7: Plots of LiDAR-derived canopy height against plant height in (a) area 1, (b) area 2, (c) area 3, and (d) combine data in three areas

\section{Discussion}

According to our method, for estimating rice plant height from laser point clouds, the relationship between measured rice plant height and LiDARderived plant height is first identified. According to the results, the LiDAR-derived plant heights in the three targeted areas are insignificantly different. However, in the last observation, the LiDARderived plant height is decreased. This result disagrees with the collected rice growth information. Downward rice leaves may cause this phenomenon by the wind generated by the propellers of UAV. For time-series data analysis, the most important is that the input data must be extracted from the same targeted area. The reflectors are used to ensure that the collected data is extracted from the same area to solve this problem. In this study, data in three targeted areas are extracted after applying many correction methods. As a result, we select successive areas within the same field with uniform growth to approximate the relationship between LiDAR-derived canopy height and plant height.

The histograms of extracted data are displayed in Figure 5. When the rice plant was small in several first observations, the histogram of point clouds must be down-skew distribution. However, the histogram peak position in the first observation was located higher than it was in the second observation. The histograms of extracted data are displayed in Figure 5. When the rice plant was small in several first observations, the histogram of point clouds must be down-skew distribution. When the rice plant is growing up, the ground surface was hidden by the rice plant; therefore, laser pulses emitted from the LiDAR sensor are fallen on rice plants themselves and could not reach the ground surface. As a result, the histograms of the rest observations were changing into a normal distribution. Moreover, the water depth of the target field was $7 \mathrm{~cm}$ in the first observation. There is a possibility that the laser pulses cannot reach the field surface because of water-filled rice paddy. As a result, in the first observation, the histogram peak shows the water level position. The histogram peak position in the first observation was located higher than in the second observation when the water was depleted. For this phenomenon, it is necessary to investigate the cause through additional experiments and data analysis in the future.

Besides, the flight altitude of the UAV itself is unstable in some cases. These conditions are related to the accuracy of estimating rice plant height. By applying Affine transformation and dividing the target area into many sub-areas with square meter size for computing LiDAR-derived canopy height, the effect of fluctuating flight is reduced. According to the results, a good correlation between measured plant height and LIDAR-derived plant height is determined (Figure 7). As mentioned above, the decrease of LiDAR-derived canopy height in the last observation on August 4 (65 DAT) might affect the relationship between LiDAR-derived canopy height and plant height.

Moreover, in the previous study (Phan et al., 2016), the last observation was performed in late July. The linear relationship between LiDARderived canopy height and plant height has been set up with a regression slope of 1,2 and $12.4 \mathrm{~cm}$ of bias value. The last observation is eliminated to ensure the accuracy of the relationship between LiDAR-derived canopy height and plant height in this study. As a result, the estimated error is improved. In detail, in the general case, the regression slope is decreased from 1.98 to 1.79 , the plant height estimation error (RMSE) is $5.5 \mathrm{~cm}$, and 
the coefficient of determination $\left(\mathrm{R}^{2}\right)$ is 0.86 . In area 1 , the results are not as good as in other areas $\left(\mathrm{RMSE}=5.4 \mathrm{~cm} ; \mathrm{R}^{2}=0.88\right.$ ). The better result was achieved with area 3 , where the flight altitude was more stable $\left(\mathrm{R}^{2}=0.90 ; \mathrm{RMSE}=4.9 \mathrm{~cm}\right)$. In area 2, the estimated results are achieved with $\mathrm{R}^{2}=0.86$ and $5.7 \mathrm{~cm}$ of RMSE. These results are acceptable and consistent with Phan et al., $2016\left(\mathrm{R}^{2} \geq 0.86\right.$; RMSE $4(\mathrm{~cm}))$ in the case study of small target areas, uniform rice growth, and the sensor's installation height was fixed. This study's steeper of regression slope might cause by the more considerable observed distance and footprint size. This result implies that our method is suitable for estimating plant height without ground surface detection from UAV- based LiDAR point cloud. The better results of RMSE in the previous study were achieved because the installation height of the LiDAR sensor was stable, and the target area was less than $1 \mathrm{~m}^{2}$.

Although a non-survey grade laser scanner is used, and the UAV altitude is unstable, the result of RMSE of $5.5 \mathrm{~cm}$ in combination is slightly inferior to our previous study and Tilly et al. (RMSE $=5$ $\mathrm{cm})$. Still, it is better than the result of Zhang and Grift $(\mathrm{RMSE}=14 \mathrm{~cm})$ and Kaizu et al. $(\mathrm{RMSE}=10$ $\mathrm{cm})$. Therefore, the possibility of wide-area estimation of rice plant height using an inexpensive UAV laser measurement system is considered.

\section{Conclusions}

In this paper, the test field was observed with HOKUYO UTM 30 LX-EW laser scanner mounted on the UAV. We try to estimate rice plant height from UAV- based LiDAR point cloud without ground surface detection. For this, the LiDARderived canopy height has calculated the difference of vertical distance of the scanning point at 95 and 1 percentile ranks in the area considered consistent growth. According to the results, and last observation is eliminated because of the poor quality of experimental data. Although the flight altitude is unstable, the LiDAR-derived canopy height correlates to plant height $\left(\mathrm{R}^{2} \geq 0.86\right)$. Based on the fieldwork results, the relational expression coefficient for converting LiDAR-derived canopy height into plant height was obtained. This coefficient might be affected by at least the cultivar, cultivation condition, degree of the foliage at the time of measurement, the arrangement of rice plant, laser incidence angle (inclination and scanning angle of the scanning surface), laser footprint diameter, the wavelength of the laser light. The effect of unstable flight altitude is reduced by analyzing the sub-area for each target area. As a result of estimating plant height using the obtained relational expression, the plant height was estimated with an error of approximately $5.5 \mathrm{~cm}$ in the whole analysis region.

Currently, the analysis region is set at several square meters. The acceptable result is achieved by doing the data processing for the sub- area of one square meter. In the future study, using the wider scanning angle as the analysis region, the larger region's information can be analyzed with one flight. In such a situation, the laser incident angles' effect must be carefully considered, and the impact of unstable flight altitude must be reduced. Moreover, other LiDAR devices can be selected for the following study. With the popularization and deployment of LiDAR in automobiles, LiDAR is becoming smaller and lower price. It will make it easier to choose a suitable LiDAR sensor to be mounted on compact UAVs. In general conclusion, this study confirms the capability of estimating plant height before the heading stage of the rice growth period over a wide area by our previous developed method to point clouds collected by the UAV-based LiDAR system.

\section{Acknowledgments}

This study was partially supported by Vietnam National University - Ho Chi Minh City under Grant number C2020-20-18 and JSPS KAKENHI Grant Number 26450362. Our sincere thanks also go to Mr. Odaka for helping us carry out the field observation. Without his precious support, it would not be possible to conduct this research.

\section{References}

Atzberger, C., 2013, Advances in Remote Sensing of Agriculture: Context Description, Existing Operational Monitoring Systems and Major Information Needs. Remote Sensing. Vol. 5, 949-981.

Badshah, M. A., Tu, N., Zou, Y. B., Ibrahim, M. and Wang, K., 2014, Yield and Tillering Response of Super Hybrid Rice Liangyoupeijiu to Tillage and Establishment Methods. Crop Journal. Vol. 2(1), 79-86.

Bai, X. F., Luo, L. J., Yan, W. H., Kovi, M. R and Xing, Y. Z., 2011, Quantitative Trait Loci for Rice Yield-Related Traits Using Recombinant Inbred Lines Derived from Two Diverse Cultivars. Genetics. Vol. 90(2), 209-215.

Bendig, J., Bolten, A., Bennertz, S., Broscheit, J., Eichfuss, S. and Bareth, G., 2014, Estimating Biomass of Barley Using Crop Surface Models (CSMs) Derived from UAV-based RGB Imaging. Remote Sensing. Vol. 6, 10395-10412. 
Bouvet, A. and Le Toan, T., 2011, Use of ENVISAT/ASAR Wide-Swath Data for Timely Rice Fields Mapping in the Mekong River Delta. Remote Sensing of Environment. Vol. 115(4), 1090-1101.

Dobermann, A. and Pampolino, M. F., 1995, Indirect Leaf Area Index Measurement as a Tool for Characterizing Rice Growth at the Field Scale. Communications in Soil Science and Plant Analysis. Vol. 26(9-10), 1507-1523.

Ehlert, D. and Heisig, M., 2005, Sources of AngleDependent Errors in Terrestrial Laser ScannerBased Crop Stand Measurement. Computers and Electronics in Agriculture. Vol. 93, 10-16.

Ehlert, D, Horn, H. J. and Adamek, R., 2006, Potential of Laser Distance Sensors for Measuring Crop Parameters. J. Applied Science. Vol. 6 (4), 898-904.

Ehlert, D., Heisig, M., and Adamek, R., 2010, Suitability of a Laser Rangefinder to Characterize Winter Wheat. Precision Agriculture. Vol. 11, 650-663.

Fablo, M. and Rembold, F., 2001, Analysis of GAC NDVI Data for Cropland Identification and Yield Forecasting in Mediterranean African Countries. Photogram. Eng. and Remote Sensing. Vol. 67(5), 593-602.

Fernandez, M. G. S., Becraft, P. W. and Yin, Y., 2009, Lübberstedt, T. From dwarves to giants: Plant height manipulation for biomass yield. Trends in Plant Science. Vol. 14(8), 454-461.

Hasanuzzaman, M., Ahamed, K. U., Nahar, K. and Akhter, N., 2010, Plant Growth Pattern, Tiller Dynamics and Dry Matter Accumulation of Wetland Rice (Oryza sativa L.) as Influenced by Application of Different Manures. Nature and Science. Vol., 8(4), 1-10.

Hosoi, F. and Omasa, K., 2006, Voxel-based 3-D Modeling of Individual Trees for Estimating Leaf Area Density Using High-Resolution Portable Scanning Lidar, IEEE Transactions on Geoscience and Remote Sensing. Vol. 44(12), 3610-3618.

Hosoi, F. and Omasa, K., 2009, Estimating Vertical Plant Area Density Profile and Growth Parameters of a Wheat Canopy at Different Growth Stages Using Three-Dimensional Portable Lidar Imaging. ISPRS Journal of Photogrammetry and Remote Sensing. Vol. 64, 151-158.

Huang, S., Fairweather, S. H., Halligan, K. Q., Fairweather, I. S., Swason, A. K. and Carbtree, R. L. A., 2009, Comparison of Individual Tree and Forest Plot Height Derived from Lidar and InSAR. Photogrammetric Engineering \& Remote Sensing. Vol. 75(2), 159-167.
Inoue, Y., Sakaiya, E. and Wang, C., 2014, Capability of C-band Backscattering Coefficients from High-Resolution Satellite SAR Sensors to Assess Biophysical Variables in Paddy Rice. Remote Sensing of Environment. Vol. 140, 257-266.

Kaizu, Y., Choi, J. M. and Kang, T. H., 2012, Grass Height and Yield Estimation Using a ThreeDimensional Laser Scanner. Environment Control in Biology. Vol. 50(1), 41-51.

Koppe, W., Gnypa, M. L., Hütt, C., Yao, Y., Miao, Y., Chen, X. and Bareth, G., 2013, Rice Monitoring with Multi-Temporal and DualPolarimetric TerraSAR-X Data. International Journal of Applied Earth Observation and Geoinformation. Vol. 21, 568-576.

Li, Y., Chen, D. and Walker, C. N. and Angus, J. F., 2010, Estimating the Nitrogen Status of Crops Using a Digital Camera. Field Crops Research. Vol. 118(3), 221-227.

Lichti, D. D., Gordon, S. J. and Stewart, M. P., 2002, Ground-based Laser Scanners: Operation, Systems, and Applications. Geomatica. Vol. 56(1), 21-33.

Lee, K. J. and Lee, B. H., 2013, Estimation of Rice Growth and Nitrogen Nutrition Status Using Color Digital Camera Image Analysis. European Journal of Agronomy. Vol. 48, 57-65.

Ma, R., 2005, DEM Generation and Building Detection from Lidar Data. Photogrammetric Engineering \& Remote Sensing. Vol. 71(7), 847-854.

Maan, G. S., Signh, C. K., Singh, M. K. and Nagarajan, B., 2015, Tree Species Biomass and Carbon Stock Measurement Using Ground Based-LiDAR. Geocarto International, Vol. 30(3). 293-310.

Mcintosh, K., Krupnik, A. and Schenk, A., 2000, Improvement of Automatic DSM Generation Over Urban Areas Using airborne Laser Scanner Data. International Archives of Photogrammetry and Remote Sensing, Vol. XXXIII, Part B3, 563-570.

Phan, A. T. T., Takahashi, K., Rikimaru, A., and Higuchi, Y., 2016, Method for Estimating Rice Plant Height without Ground Surface Detection Using Laser Scanner Measurement. Journal of Applied Remote Sensing. Vol. 10(4). http://dx.doi.org/10.1117/1.JRS.10.046018.

Rice, S. K., Gutman, C., and Krouglicof, N., 2005, Laser Scanning Reveals Bryophyte Canopy Structure. The New Phytologist. Vol. 166(2), 695-704. http://www.jstor.org/stable/1514711.

Sakamoto, T., Shibayama, M., Kimura, A. and Tadaka, E., 2011, Assessment of Digital Camera-Derived Vegetation Indices in 
Quantitative Monitoring of Seasonal Rice Growth. ISPRS Journal of Photogrammetry and Remote Sensing. Vol. 66(6), 872-882.

Shao, Y., Fan, X., Liu, H., Xiao, J., Ross, S., Brisco, B. and Brown, R., 2001, Staples, G. Rice Monitoring and Production Estimation Using Multitemporal RADARSAT. Remote Sensing of Environment. Vol. 76(3), 310-325.

Sibona, E., Vitali, A., Meloni, F., Caffo, L., Dotta, A., Lingua, E., Motta, R. and Garbarino, M., 2017, Direct Measurement of Tree Height Provides Different Results on the Assessment of LiDAR Accuracy. Forests. Vol., 8(7), 12 pages ; doi:10.3390/f8010007.

Singh, B., Singh, V., Singh, Y., Thind, H. S., Kumar, A., Gupta, R. K., Kaul, A. and Vashistha, M., 2012, Fixed-Time Adjustable Dose Site-Specific Fertilizer Nitrogen Management in Transplanted Irrigated Rice (Oryza Sativa L.) in South Asia. Field Crops Research. Vol. 126, 63-69.

Swain, K. C., and Zaman, Q. U., 2012, Rice Crop Monitoring with Unmanned Helicopter Remote Sensing Images. Remote Sensing of Biomass Principles and Applications, Vol. 253-272.

Takai, T., Matsuura, S., Nishio, T., Ohsumi, A., Tatsuhiko, S. and Horie, T., 2006, Rice Yield Potential is Closely Related to Crop Growth Rate During Late Reproductive Period. Field Crops Research. Vol. 96, 328-335.

Tennakoon, S. B., Murty, V. V. N. and Eiumnoh, A., 1992, Estimation of Cropped Area and Grain Yield of Rice Using Remote Sensing Data. Intl. J. Remote Sensing. Vol. 13(3), 427-439.

Thomas, J. B., Schaalje, G. B. and Grant, M. N., 1994, Height, Competition and Yield Potential in Winter Wheat. Euphytica. Vol. 74, 9-17.

Tilly, N., Hoffmeister, D., Cao, Q., Huang, S., LenzWiedemann, V., Miao, Y. and Bareth, G., 2014, Multitemporal Crop Surface Models: Accurate Plant Height Measurement and Biomass Estimation with Terrestrial Laser Scanning in Paddy Rice. Journal of Applied Remote Sensing. Vol. 8, 1-22.

Yamamoto, Y., Kawaguchi, S. and Takahashi, W., 1994, Application of Developmental Model for Analysis of Growth and Development of Rice Transplanted in different Plant Numbers Per Hill. Japanese Journal of Crop Science. Vol. 63(2), 208-214.

Wang, F., Cheng, F. M. and Zhang, G. P., 2007, Difference in Grain Yield and Quality among Tillers in Rice Genotypes Differing in Tillering Capacity. Rice Science. Vol. 14, 135-140.
Wang, C. and Glenn, N. F., 2008, A Linear Regression Method for Three Canopy Height Estimation Using Airborne LIDAR Data. Can. J. Remote Sensing. Vol. 34(2), 217-227.

Wang, Y., Wang, D., Zhang, G. and Wang, J., 2013, Estimating Nitrogen Status of Rice Using the Image Segmentation of G-R Thresholding Method. Field Crops Research. Vol. 149, 33-39.

Willers, J., 2012, The Illuminating Role of Laser Scanning Digital Elevation Models in Precision Agriculture Experimental Designs - An AgroEcology Perspective, Laser Scanner Technology, Dr. J. Apolinar Munoz Rodriguez (Ed.), InTech. DOI: 10.5772/35197.

Zawawi, A. A., Shiba, M. and Jemali, N., 2015, Accuracy of LiDAR-based Tree Height Estimation and Crown Recognition in a Subtropical Evergreen Broad-Leaved Forest in Okinawa, Japan. Forest Systems. Vol. 24(1), e002, 11 pages. http://dx.doi.org/10.5424/fs/2015241-05476.

Zhang, L. and Grift T. E., 2012, A LIDAR-based Crop Height Measurement System for Miscanthus Giganteus. Computers and Electronics in Agriculture. Vol. 85, 70-76. 